\title{
FAP Regimen
}

National Cancer Institute

\section{Source}

National Cancer Institute. FAP Regimen. NCI Thesaurus. Code C63398.

A reg imen containing fluorouracil, doxorubicin and cisplatin used for the treatment of advanced-stage gastric cancer. 